04

\title{
Поперечная электрическая проводимость и диэлектрическая проницаемость поликристаллического металла
}

\author{
(C) Лам Тхи Ньюнг, А.А. Юшканов \\ Московский государственный областной университет, \\ Мытищи, Московская обл., Россия \\ e-mail: nhunglam279@gmail.com, yushkanov@inbox.ru
}

Поступило в Редакцию 23 декабря 2020 г.

В окончательной редакции 23 декабря 2020 г.

Принято к публикации 26 января 2021 г.

Рассмотрено поведение вырожденной электронной плазмы под действием переменного поперечного электрического поля слабой интенсивности. Расмотрено линеаризованное кинетическое уравнение для электронов в поликристаллическом металле. Это кинетическое уравнение учитывает рассеяние электронов на границах кристаллитов поликристаллического металла. На основе этого уравнения получено выражение для поперечной проводимости и поперечной диэлектрической проницаемости поликристаллического металла.

Ключевые слова: кинетическое уравнение, металл, поперечная проводимость, поперечная диэлектрическая проницаемость.

DOI: 10.21883/JTF.2021.06.50863.122-20

\section{Введение}

В настоящее время наблюдается растущий интерес к электромагнитным свойствам поликристаллического металла [1-10]. Обычно для анализа проблемы используется приближение, разработанное в [11]. При этом вклады в процессы, обусловленные рассеянием электронов на примесях и фононах и на границах зерен поликристаллического металла, учитываются путем их формального сложения. Однако такой подход не применим для описания сложных кинетических процессов, при которых пространственная и временна́я зависимости функции распределения нетривиальны. Это касается, в частности, диэлектрической проницаемости электронного газа с учетом временно́й и пространственной дисперсий.

Такая ситуация характерна для изучения диэлектрических свойств поликристаллического металла. При этом необходим подход, при котором различные вклады в кинетические процессы учитывались бы единым образом. Такой подход рассмотрен в настоящей работе.

Рассматривается кинетическое уравнение для вырожденной электронной плазмы. Поле считается достаточно слабым для возможности линеаризации имеющихся уравнений. При этом будет рассмотрен случай, когда электроны после рассеяния в той или иной степени сохраняют информацию о своей первоначальной скорости. Это происходит при учете электрон-электронного рассеяния, а так же при рассмотрении сильно гранулированных сред. В последнем случае существенно рассеяние электронов на границе зерен, когда требуется учесть эффект отражения электронов от этих границ.

Линеаризованное кинетическое уравнение для вырожденного электронного газа широко используется для исследования различных явлений в металлах. Например, для исследования скин-эффекта [12-17], для ис- следования электрических свойств металлических пленок [18-20]. Также линеаризованное кинетическое уравнение используется для описания колебания электронной плазмы под действием электрического поля $[21,22]$.

\section{1. Кинетическое уравнение для поликристаллического металла}

Рассмотрим кинетическое уравнение для электронов в $\mathbf{v}, \tau$-приближении для функции распределения электронов $f(\mathbf{v}, \mathbf{r}, t)[12,23-25]$

$$
\frac{\partial f}{\partial t}+\mathbf{v} \frac{\partial f}{\partial \mathbf{r}}+e \mathbf{E} \frac{\partial f}{\partial \mathbf{p}}=v\left(f_{e q}-f\right) .
$$

Здесь $\mathbf{v}, e-$ скорость и заряд электронов, $v-$ частота рассеяния электронов, $v=1 / \tau, \tau$ - среднее время свободного пробега электронов, $\mathbf{E}-$ напряженность электрического поля.

Функция $f_{e q}$ описывает локально равновесное распределение вырожденного Ферми-газа электронов:

$$
f_{e q}(\mathbf{v}, \mathbf{r}, t)=\Theta[\mu(\mathbf{r}, t)-\mathscr{E}(\mathbf{v}-\mathbf{u}(\mathbf{r}, t))] .
$$

Здесь

$$
\mathscr{E}(\mathbf{v}-\mathbf{u}(\mathbf{r}, t))=\frac{m}{2}[\mathbf{v}-\mathbf{u}(\mathbf{r}, t)]^{2},
$$

$\mu(\mathbf{r}, t)$ - химический потенциал электронного газа. Функция $\mathbf{u}(\mathbf{r}, t)$ учитывает тот факт, что равновесная скорость электронного газа при наличии явлений переноса может быть отлична от нуля, $\Theta(x)$ - функция Хэвисайда,

$$
\Theta(x)= \begin{cases}1, & x>0 \\ 0, & x<0\end{cases}
$$


При этом считается, что поверхность Ферми металла имеет сферическую форму.

Однако в общем случае после рассеяния средняя скорость электрона будет отлична от нуля. То есть электрон будет „помнить“ о скорости, которую от имел до рассеяния.

В случае, когда доминирует электрон-электронное рассеяние, электрон после рассеяния приобретает в среднем скорость, пропорциональную исходной, хотя и меньшую по абсолютной величине.

Будем предполагать, что равновесная скорость электронов после рассеяния и пропорциональна средней скорости электронов $\langle\mathbf{v}\rangle$ :

$$
\mathbf{u}=\beta\langle\mathbf{v}\rangle .
$$

В величине $\beta$ учитывалось то, что электроны рассеяния частично сохраняют свою память о своей скорости до рассеяния. В данном подходе величина $\beta$ является эмпирическим коэффициентом.

Близкий к подобному подход ранее использовался для учета электрон-электронных столкновений [26-28]. В случае электрон-электронных столкновений величина $\beta$ положительна, так как рассеяние электронов происходит преимущественно вперед. В случае рассеяния электронов на границе кристаллитов поликристаллического металла величина $\beta$ отрицательна, т.е. $\beta<0$. Это связано с тем, что в данном случае рассеяние электронов происходит преимущественно в направлении, противоположном направлению первоначального движения электронов.

Пусть электрическое поле изменяется по закону

$$
E_{y}=E_{0 e}^{i(k x-\omega t)}, \quad E_{x}=E_{z}=0 .
$$

Рассмотрим, какой будет проводимость электронного газа в этом случае. Такая проводимость называется поперечной электронной проводимостью $\sigma^{\mathrm{tr}}$.

В этом случае химический потенциал электронного газа будет постоянным.

Рассмотрим линеаризацию кинетического уравнения (1) в этом случае.

Равновесную функцию распределения линеаризуем относительно абсолютного (независящего от координат) распределения Ферми

$$
f_{F}(\mathbf{v}) \equiv f_{0}(\mathbf{v})=\Theta\left[\mu_{0}-\mathscr{E}(\mathbf{v})\right]
$$

При линеаризации локально равновесной функции распределения получаем:

$$
f_{e q}(\mathbf{v})=f_{0}(\mathbf{v})+\delta\left(\mathscr{E}_{F}-\mathscr{E}\right) m \mathbf{v u}(\mathbf{r}, t)
$$

Здесь $\delta(x)$ - дельта-функция Дирака.

Для вырожденного Ферми-газа электронов в металле

$$
\mu_{0}=\mathscr{E}_{F}=\frac{m v_{F}^{2}}{2}=\frac{p_{F}^{2}}{2 m}, \quad \mathscr{E}(\mathbf{v})=\frac{m v^{2}}{2},
$$

$v_{F}$ - скорость электронов на поверхности Ферми, которая считается сферической, $\mathscr{E}_{F}-$ энергия электронов на поверхности Ферми, $\mathscr{E}(\mathbf{v})-$ кинетическая энергия электронов.

Для вырожденного Ферми-газа электронов имеем

$$
\begin{aligned}
& \delta\left(\mathscr{E}-\mathscr{E}_{F}\right)=\delta\left[\frac{m \mathbf{v}^{2}}{2}-\frac{m \mathbf{v}_{F}^{2}}{2}\right] \\
& =\delta\left(\frac{m}{2}\left(v-v_{F}\right)\left(v+v_{F}\right)\right)=\frac{1}{m v_{F}} \delta\left(v-v_{F}\right) \\
& =\frac{1}{m v_{F}^{2}} \delta\left(\frac{v}{v_{F}}-1\right)=\frac{1}{2 \mathscr{E}_{F}} \delta\left(\frac{v}{v_{F}}-1\right) .
\end{aligned}
$$

Импульс электрона равен $\mathbf{p}=m \mathbf{v}$. Соответственно на поверхности Ферми $p_{F}=m v_{F}$.

Введем безразмерный импульс (скорость) электронов

$$
\mathbf{P}=\frac{\mathbf{p}}{p_{F}}=\frac{\mathbf{v}}{v_{F}} .
$$

Тогда

$$
\delta\left(\mathscr{E}-\mathscr{E}_{F}\right)=\frac{1}{2 \mathscr{E}_{F}} \delta\left(\frac{v}{v_{F}}-1\right)=\frac{1}{2 \mathscr{E}_{\mathscr{F}}} \delta(P-1),
$$

и линеаризованная локально равновесная функция распределения записывается в виде

$$
f_{e q}=\Theta\left(1-P^{2}\right)+\delta(P-1) \mathbf{P U}(\mathbf{r}, t) .
$$

Здесь $\mathbf{U}$ - безразмерная равновесная скорость электронов

$$
\mathbf{U}=\frac{\mathbf{u}}{v_{F}}
$$

Плотность электрического тока $\mathbf{j}(\mathbf{r}, t)$ можно представить в виде

$$
\mathbf{j}(\mathbf{r}, t)=e \int f \mathbf{v} \frac{2 d^{3} p}{(2 \pi \hbar)^{3}} .
$$

Равновесная концентрация электронов $N$ находится по формуле

$$
\int f_{0} \frac{2 d^{3} p}{(2 \pi \hbar)^{3}}=N
$$

При этом

$$
N=\frac{8 \pi p_{F}^{3}}{3(2 \pi \hbar)^{3}} .
$$

Данное соотношение позволяет найти связь между равновесной концентрацией электронов $N$ и величиной импульса электрона на поверхности Ферми $p_{F}$.

В линейном приближении функцию распределения электронов можно искать в виде

$$
f=\Theta\left(1-P^{2}\right)+h(\mathbf{r}, \mathbf{P}, t) \delta(P-1) .
$$

Здесь $f_{0}(P)=\Theta\left(1-P^{2}\right)$ - вырожденная статистика. 
С помощью (5) запишем кинетическое уравнение (1) в виде

$$
\begin{aligned}
& \frac{\partial f}{\partial t}+v_{F} \mathbf{P} \frac{\partial f}{\partial \mathbf{r}}+\frac{e}{m v_{F}} \mathbf{E}(\mathbf{r}, t) \frac{\partial f}{\partial \mathbf{P}} \\
& =v \delta(P-1)[\mathbf{P U}(\mathbf{r}, t)-h(\mathbf{r}, \mathbf{P}, t)] .
\end{aligned}
$$

Очевидно:

$$
\frac{\partial f_{0}}{\partial t}=\frac{\partial f_{0}}{\partial \mathbf{r}}=0
$$

В линейном приближении по электрическому полю можно записать

$$
\mathbf{E}(\mathbf{r}, t) \frac{\partial f}{\partial \mathbf{P}}=\mathbf{E}(\mathbf{r}, t) \frac{\partial f_{0}}{\partial \mathbf{P}}=-\mathbf{P E}(\mathbf{r}, t) \delta(P-1) .
$$

При выводе этого уравнения было учтено, что

$$
\frac{\partial f_{0}}{\partial \mathbf{P}}=-\mathbf{P} \delta(P-1)
$$

Учитывая эти соотношения, кинетическое уравнение (6) можно переписать в следующем виде:

$$
\begin{aligned}
& \frac{\partial h}{\partial t}+v_{F} \mathbf{P} \frac{\partial h}{\partial \mathbf{r}}-\frac{e}{m v_{F}} \mathbf{P E}(\mathbf{r}, t) \\
& =v[\mathbf{P U}(\mathbf{r}, t)-h(\mathbf{r}, \mathbf{P}, t)] .
\end{aligned}
$$

Уравнение (7) преобразуем к виду

$$
\begin{aligned}
\frac{\partial h}{\partial t} & +v_{F} \mathbf{P} \frac{\partial h}{\partial \mathbf{r}}-\left(\frac{e}{m v_{F}} \mathbf{E}(\mathbf{r}, t)+v \mathbf{U}(\mathbf{r}, t)\right) \mathbf{P} \\
& =-v h(\mathbf{r}, \mathbf{P}, t) .
\end{aligned}
$$

Ищем решение в виде

$h=e^{i(k x-\omega t)} H, \quad U_{y}=e^{i(k x-\omega t)} W, \quad j_{y}=e^{i(k x-\omega t)} j$.

Подствляя эти выражения в уравнение (8), получаем

$$
-i \omega H+i k v_{F} P_{x} H-\frac{e}{m v_{F}}\left(E_{0}+\frac{v m v_{F}}{e} W\right) P_{y}=-v H .
$$

Отсюда следует, что плотность тока $j$ можно представить в следующем виде:

$$
j=\sigma_{0}^{\mathrm{tr}}\left(E_{0}+\frac{\nu m v_{F}}{e} W\right) .
$$

Здесь $\sigma_{0}^{t r}$ поперечная проводимость в случае, когда рассеяние на границах зерен отсутствует, т. е. $\beta=0$ так как $W \sim \beta$.

Ho

$$
W=\frac{\beta j}{e N v_{F}} .
$$

Тогда из уравнения (9) получаем

$$
j=\sigma_{0}^{\operatorname{tr}}\left(E_{0}+\frac{\beta v m}{e^{2} N} j\right) .
$$

Отсюда следует

$$
j=\frac{\sigma_{0}^{\operatorname{tr}}}{\left(1-\frac{\beta v m}{e^{2} N} \sigma_{0}^{\operatorname{tr}}\right)} E_{0} .
$$

По определению

$$
\sigma^{\operatorname{tr}}=\frac{j_{y}}{E_{y}}=\frac{j}{E_{0}}
$$

Поэтому последнюю формулу можно переписать в виде

$$
\sigma^{\operatorname{tr}}=\frac{\sigma_{0}^{\operatorname{tr}}}{\left(1-\frac{\beta \sigma_{0}^{\mathrm{tr}}}{\sigma}\right)}
$$

Здесь $\sigma=e^{2} N /(v m)$ - статическая проводимость электронного газа.

При $\beta=0$ получаем $\sigma^{\text {tr }}=\sigma_{0}^{\text {tr }}$. Таким образом, полученный результат переходит в ранее известный для поперечной проводимости при отсутствии рассеяния на границах зерен.

По мере того как параметр $\beta$ растет по модулю (оставаясь отрицательным), величина $\sigma^{\text {tr }}$ уменьшается. Это связано с тем, что границы зерен увеличения по модулю параметра $\beta$ становятся все менее проницаемыми и рассеяние электронов в направлении, противоположном исходному, все более доминирует.

Для случая отсутствия рассеяния электронов на границах зерен, т. е. когда $\beta=0$, имеем

$$
-i \omega H+i k v_{F} P_{x} H-\frac{e}{m v_{F}} E_{0} P_{y}=-v H .
$$

Отсюда находим функцию $H$

$$
H=\frac{e P_{y}}{m v_{F}\left(v-i \omega+i k v_{F} P_{x}\right)} E_{0} .
$$

Выражение для плотности тока имеет вид

$$
j=e N \int v_{y} H \delta(P-1) \frac{2 d^{3} p}{(2 \pi \hbar)^{3}} .
$$

Учитывая соотношение (11), получаем

$$
j=\frac{3 e^{2} N E_{0}}{4 \pi m} \int \frac{P_{y}^{2}}{\left(v-i \omega+i k v_{F} P_{x}\right)} \delta(P-1) d^{3} P .
$$

Данное выражение можно преобразовать к виду

$$
j=\frac{3 e^{2} N E_{0}}{4 m} \int_{-1}^{1} \frac{1-t^{2}}{v-i \omega+i k v_{F} t} d t .
$$

В случае $k=0, \omega=0$ получаем:

$$
\sigma_{0}^{\operatorname{tr}}=\sigma=\frac{e^{2} N}{v m}
$$

Это стандартное выражение для статической проводимости электронного газа. 
В общем случае для $\sigma_{0}^{\text {tr }}$ получаем следующее выражение:

$$
\sigma_{0}^{\mathrm{tr}}=\frac{3 e^{2} N}{4 m} \int_{-1}^{1} \frac{1-t^{2}}{v-i \omega+i k v_{F} t} d t .
$$

Поперечная диэлектрическая проницаемость описывается соотношением [24]

$$
\varepsilon_{\mathrm{tr}}=1+\frac{4 \pi i}{\omega} \sigma_{\mathrm{tr}}
$$

Поведение плазмы характеризует величина $\omega_{p}$ - плазменная (ленгмюровская) частота. Тогда $\omega_{p}^{2}=4 \pi e^{2} N / m, \sigma=\omega_{p}^{2} /(4 \pi v)$.

Можно ввести безразмерную частоту $\Omega=\omega / \omega_{p}$ и безразмерную частоту столкновений $\epsilon=v / \omega_{p}$. А также безразмерное волновое число $\kappa=k v_{F} / \omega_{p}$.

Тогда получаем

$$
\sigma_{0}^{\mathrm{tr}}=\frac{3 \omega_{p}}{16 \pi} \int_{-1}^{1} \frac{1-t^{2}}{\epsilon-i \Omega+i \kappa t} d t .
$$

Это выражение можно представить в виде

$$
\sigma_{0}^{\mathrm{tr}}=\frac{3 \omega_{p}}{16 \pi} \Sigma_{0}^{\mathrm{tr}}, \quad \Sigma_{0}^{\mathrm{tr}}=\int_{-1}^{1} \frac{1-t^{2}}{\epsilon-i \Omega+i \kappa t} d t .
$$

Отметим, что $\Sigma_{0}^{\mathrm{tr}}-$ безразмерная величина.

$$
\begin{aligned}
\sigma^{\mathrm{tr}} & =\frac{\sigma_{0}^{\mathrm{tr}}}{\left(1-\frac{\beta \nu m}{e^{2} N} \sigma_{0}^{\mathrm{tr}}\right)}=\frac{\sigma_{0}^{\mathrm{tr}}}{\left(1-\frac{4 \pi \beta v}{\omega_{p}^{2}} \sigma_{0}^{\mathrm{tr}}\right)} \\
& =\frac{\sigma_{0}^{\mathrm{tr}}}{\left(1-\frac{3 \beta v}{4 \omega_{p}} \Sigma_{0}^{\mathrm{tr}}\right)} .
\end{aligned}
$$

Отсюда получаем

$$
\sigma^{\mathrm{tr}}=\frac{3 \omega_{p}}{16 \pi} \frac{\Sigma_{0}^{\mathrm{tr}}}{\left(1-\frac{3 \beta \epsilon}{4} \Sigma_{0}^{\mathrm{tr}}\right)} .
$$

Перепишем это выражение в виде

$$
\begin{aligned}
\sigma^{\mathrm{tr}} & =\frac{3 \omega_{p}}{16 \pi} \Sigma_{0}^{\mathrm{tr}} \Sigma_{\mathrm{tr}}, \\
\Sigma_{\mathrm{tr}} & =\frac{1}{\left(1-\frac{3 \beta \epsilon}{4} \Sigma_{0}^{\mathrm{tr}}\right)} .
\end{aligned}
$$

Поперечная диэлектрическая проницаемость $\varepsilon_{\mathrm{tr}}$ следующим образом выражается через поперечную электрическую проводимость $\Sigma_{\text {tr }}[24]$

$$
\begin{gathered}
\varepsilon_{\mathrm{tr}}=1+\frac{4 \pi i}{\omega} \sigma^{\mathrm{tr}}=1+\frac{3 i}{4 \Omega} \Sigma^{\mathrm{tr}}, \\
\Sigma^{\mathrm{tr}}=\Sigma_{0}^{\mathrm{tr}} \Sigma_{\mathrm{tr}} .
\end{gathered}
$$

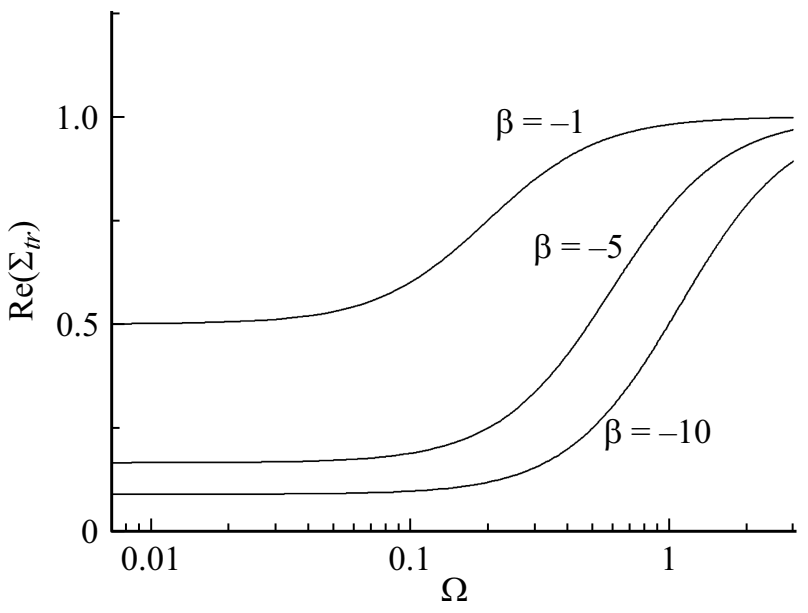

Рис. 1. Зависимость действительной части безразмерной поперечной проводимости $\Sigma_{\mathrm{tr}}$ от безразмерной частоты $\Omega$. Здесь $\epsilon=0.1, \kappa=0.01$.

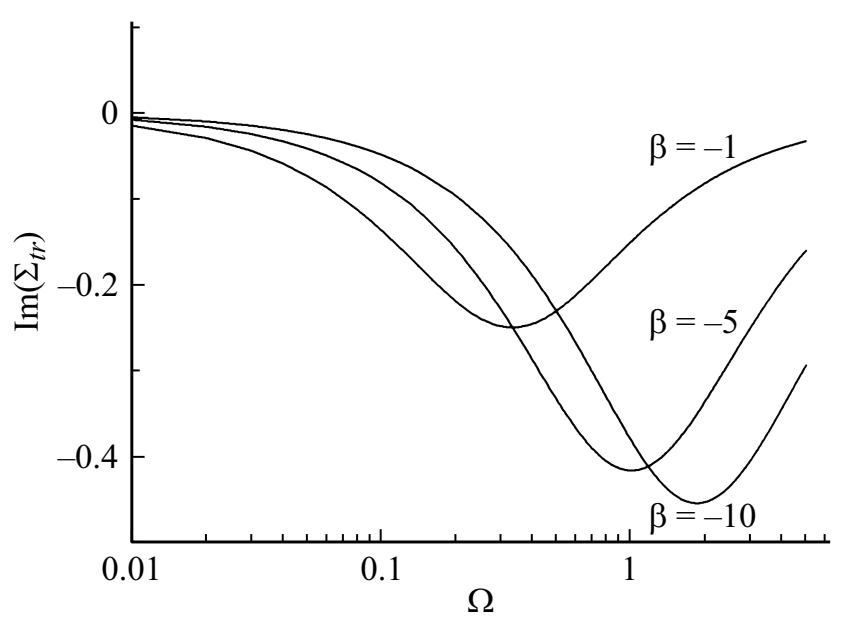

Рис. 2. Зависимость мнимой части безразмерной поперечной проводимости $\Sigma_{\text {tr }}$ от безразмерной частоты $\Omega$. Здесь $\epsilon=0.1$, $\kappa=0.01$.

На рис. 1 и 2 представлены графики зависимости действительной и мнимой части безразмерной поперечной электрической проводимости $\Sigma_{\mathrm{tr}}$ от безразмерной частоты $\Omega$. Из рис. 1 видно, что действительная часть безразмерной поперечной электрической проводимости $\Sigma_{\text {tr }}$ стремится к единице с ростом безразмерной частоты $\Omega$. Это связано с тем, что при росте частоты $\Omega$ величина $\Sigma_{0}^{\mathrm{tr}} \sim 1 / \Omega$ стремится к нулю. При этом $\Sigma_{\mathrm{tr}} \rightarrow 1$. Так что в этом пределе $\operatorname{Re}\left(\Sigma_{\text {tr }}\right) \rightarrow 1$, а $\operatorname{Im}\left(\Sigma_{\text {tr }}\right) \rightarrow 0$. Это происходит при всех значениях величины $\beta$.

Tо, что $\operatorname{Im}\left(\Sigma_{\text {tr }}\right) \rightarrow 0$ при $\Omega \rightarrow \infty$ и при $\Omega \rightarrow 0$ проявляется на характере кривых на рис. 2 .

На рис. 3 и 4 приведены графики зависимости действительной и мнимой части безразмерной поперечной электрической проводимости $\Sigma_{\mathrm{tr}}$ от безразмерной величины $\kappa$ при различных частотах $\Omega$. При этом параметр $\beta$ был принят равным -5 . Из графиков видно, что мнимая и действительная части безразмерной поперечной элек- 


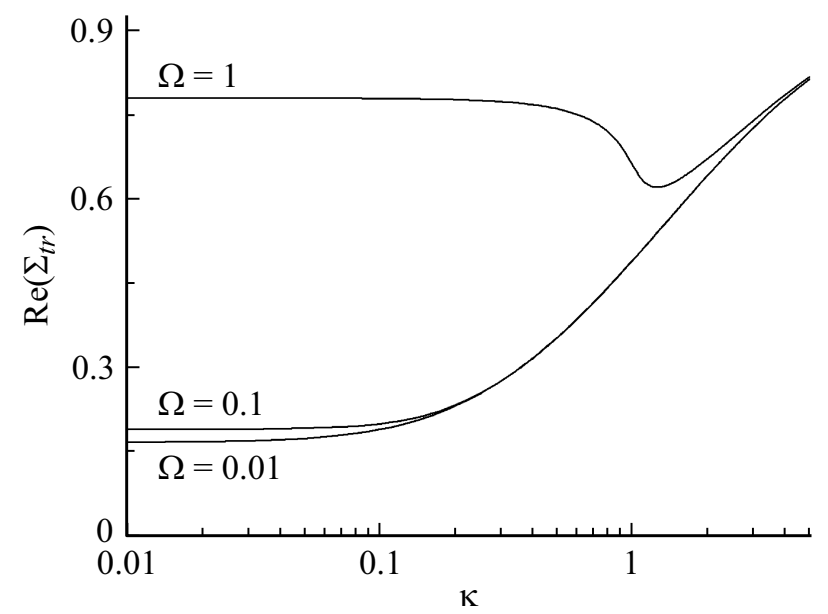

Рис. 3. Зависимость действительной части безразмерной поперечной проводимости $\Sigma_{\mathrm{tr}}$ от безразмерной величины $\kappa$. Здесь $\epsilon=0.1, \beta=-5$.

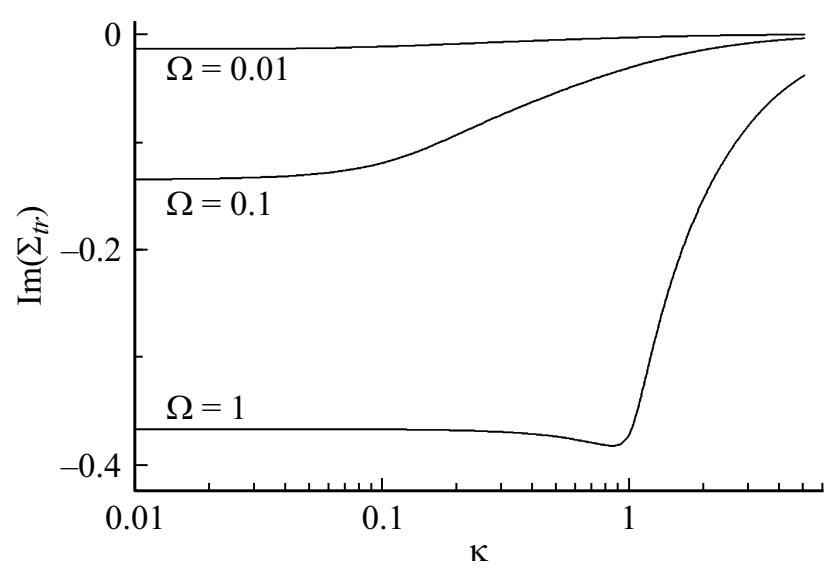

Pис. 4. Зависимость мнимой части безразмерной поперечной проводимости $\Sigma_{\text {tr }}$ от безразмерной частоты $\Omega$. Здесь $\epsilon=0.01$, $\beta=-5$.

трической проводимости имеют существенно различную зависимость от величины $\Omega$ при различных значениях параметра $\kappa$.

\section{Заключение}

Получены выражения для поперечной электрической проводимости и поперечной диэлектрической проницаемости поликристаллического металла. При этом проведен учет возможности того, что после столкновения электроны частично сохраняют информации о своей скорости до сстолкновения. Такая ситуация возникает при рассеянии электронов на границе раздела кристаллитов. Проведено графическое исследование полученных результатов. Показано, что учет зависимости поперечной электрической проводимости и поперечной диэлектрической проницаемости поликристаллического металла от рассеяния электронов на границах зерен оказывает существенное влияние на значения этих величин и частот- ные зависимости. При некоторых значениях параметров зависимость поперечной электрической проводимости от волнового числа $\kappa$ становится немонотонной.

Рассмотренное кинетическое уравнение может быть использовано при изучении проводимости тонких металлических проволок и пленок. Оно также может быть применено для исследования скин-эффекта в поликристаллическом металле и при анализе взаимодействия электромагнитного излучения с тонкими металлическими пленками.

\section{Конфликт интересов}

Авторы заявляют, что у них нет конфликта интересов.

\section{Список литературы}

[1] J.S. Chawla, F. Gstrein, K.P. O’Brien, J.S. Clarke, D. Gall. Phys. Rev. B, 84, 235423 (2011).

[2] S. Canulescu, C.N. Borca, K. Rechendorff, S. Davipsdyttir, K. Pagh Almtoft, L.P. Nielsen, J. Schou. Appl. Phys. Lett., 108, 141909 (2016).

[3] B. Feldman, S. Park, M. Haverty, S. Shankar, S Dunham. Physica Status Solidi B, 247 (7), 1791 (2010).

[4] A.K. Majee, C.J. Foss, Z. Aksamija. Sci. Rep., 7, 16597 (2017).

[5] S.K. Mandal, T.K. Nath, V.V. Rao. J. Phys: Condens Matter., 20 (38), 385203 (2008).

[6] M.Z. Liu, K.W. Xu. J. Mater. Res., 23 (6), 1658 (2008).

[7] S.W. Ng, K.P. Lim, S.A. Halim, H. Jumiah. Res. Phys., 9, 1192 (2018).

[8] N, Mikhuri, V. Agarwal, A. Gaur, H.K. Singh. J. Rare Earth, 29, 654 (2011).

[9] C. Durkan, M.E. Welland. Phys. Rev. B, 61, 14215 (2000).

[10] W. Xue, W. Gua. AIP Adv., 6, 115001 (2016).

[11] A.F. Mayadas, M. Shatzkes, J.F. Janak. Appl. Phys. Lett., 14 (11), 345 (1969).

[12] А.А. Абрикосов Основы теории металла (Наука, М. 1977)

[13] G.E.H. Reuter, E.H. Sondheimer. Proc. Roy. Soc., 195, 336 (1948).

[14] K.L. Kliewer, R. Fuchs. Phys. Rev., 172 (3), 607 (1968).

[15] А.В. Латышев, А.А. Юшканов. Ж. выч. матем. и матем. физики, 39 (6), 989 (1999).

[16] А.В. Латышев, А.А. Юшканов. ЖТФ, 70 (8), 1 (2000).

[17] А.В. Латышев, А.А. Юшканов. ФТТ, 51 4, 630 (2009).

[18] А. Паредес-Хуарес, Ф. Диас-Монхе, Н.М. Макаров, Ф. Перес-Родригес. Письма в ЖЭТФ, 90 6, 687 (2009).

[19] A.A. Yushkanov, N.V. Zverev. Phys. Lett. A, 381, 679 (2017).

[20] И.А. Кузнецова, О.В. Савенко, А.А. Юшканов. ЖТФ, 87 (12), 1769 (2017).

[21] Ф. Платцман., П. Вольф. Волны и взаимодействие в плазме твердых тел (Мир, М. 1975), 436 с.

[22] А.В. Латышев, А.А. Юшканов. ФТТ, 48 (12), 2113 (2006).

[23] Е.М. Лифшиц, Л.П. Питаевский. Физическая кинетика (Наука, М. 1979)

[24] В.П. Силин, А.А. Рухадзе. Электромагнитные свойства плазмы и плазмоподобных сред (Госатомиздат, М. 1961), $244 \mathrm{c}$.

[25] E.H. Sondheimer. Adv. Phys., 50 (6), 499 (2010).

[26] E. Borchi, S. De Gennaro. J. Phys. F, 10, 271 (1980).

[27] S. De Gennaro, A. Rettory. J. Phys. F, 14, 237 (1984).

[28] S. De Gennaro, A. Rettory. J. Phys. F, 15, 227 (1985). 